\section{Fatal lung abscess due to Lactobacillus casei ss rhamnosus}

\author{
S S Namnyak, A L T Blair, D F Hughes, \\ P McElhinney, M R Donnelly, J Corey
}

\begin{abstract}
A fatal case of community acquired pneumonia due to Lactobacillus casei ss rhamnosus is reported. Clinicians should be aware of this type of pneumonia.
\end{abstract}

Bronchopulmonary infections due to Lactobacillus casei ss rhamnosus are rarely reported, with only one previous case ${ }^{1}$ described worldwide. We report the clinical, microbiological, and histopathological features of a patient who developed a fatal lung abscess due to this organism.

\section{Case report}

A retired 73 year old male security worker was admitted to Tyrone County Hospital in August 1990. He presented with a two week history of chest pain, nausea and vomiting, malaise, dyspnoea on exertion, and cough with yellow sputum. He was a longstanding heavy smoker, and had developed emphysema.

His general practitioner had treated him with broad spectrum antibiotics for two weeks but he deteriorated and was admitted to hospital. His blood pressure was then $130 / 70 \mathrm{~mm} \mathrm{Hg}$, pulse rate $85 / \mathrm{min}$, temperature $37.5^{\circ} \mathrm{C}$, and respiratory rate $22 / \mathrm{min}$. He had right pulmonary basal crepitations. The erythrocyte sedimentation rate was $70 \mathrm{~mm}$ in one hour (Westergren), and the white blood cell count $15.9 \times 10^{9} / 1$ (92\% neutrophils). The sputum was thick and purulent and had a foul odour. Microscopic examination of the sputum showed numerous pus cells and Gram positive bacilli and moderate amounts of yeasts. No acid fast bacilli were seen. The admission chest radiograph (fig 1) showed consolidation and cavitation in the right apex and a small area of consolidation of the right costophrenic angle. The left lung was clear.

Daily sputum cultures were subcultured on blood agar plates (incubated aerobically and anaerobically (GasPak), chocolate plates (incubated in $5-7 \%$ carbon dioxide), and Sabouraud's and MacConkey plates (incubated in air). All plates were incubated at $37^{\circ} \mathrm{C}$ for 72 hours initially, and reincubated (except MacConkey) for one week. The sputum cultures yielded a heavy growth of Gram positive bacilli ("diphtheroid") after three days' incubation. The organisms grew aerobically, but growth was more confluent anaerobically in the presence of carbon dioxide. Antibiotic disc

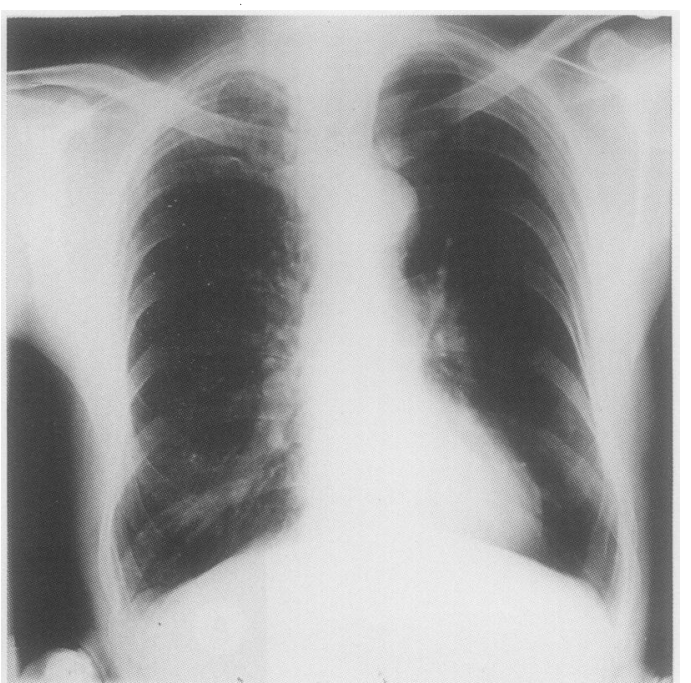

Figure 1 Chest radiograph at admission showing consolidation in the right apex and a small area of consolidation at the right costophrenic angle.

diffusion tests were carried out under anaerobic conditions (metronidazole) and with an increased carbon dioxide concentration in accordance with techniques for testing slow growing and anaerobic microorganisms. ${ }^{2}$ An overnight DST (diagnostic sensitivity test) broth was diluted to achieve a semi-confluent growth and swabbed on to DST plates impregnated with antibiotic discs and incubated at $37^{\circ} \mathrm{C}$ in $5-7 \%$ carbon dioxide and anaerobically (metronidazole) for 48 hours. The organisms were sensitive to ampicillin, tetracycline, gentamicin, ciprofloxacin, and mezlocillin, but resistant to penicillin, trimethoprim, cephradine, cefotaxime, and flucloxacillin. Sensitivity testing for metronidazole gave an equivocal result. Candida albicans and Aspergillus fumigatus were also isolated. Multiple blood cultures yielded no growth. Serological tests for respiratory viruses, chlamydia, Coxiella barnetii, mycoplasma, and legionella gave negative results, as did latex agglutination tests for pneumococcal antigen in blood, serum, sputum, and urine.

The patient was treated empirically with gentamicin and ampicillin for three days and, once the results of sensitivity testing were known, for a further five days. His condition deteriorated, however; his sputum became more copious and purulent, the white blood cell count rose to $25.4 \times 10^{9} / 1$ (95\% neutrophils), and his temperature rose to $39^{\circ} \mathrm{C}$. A chest radiograph on day 8 revealed extensive consolidation in the right upper and mid zones with relative sparing of the right lower zone (fig 2). In view of the known discrepancy between in vitro sensitivity and clinical response to treatment in $L$ casei ss rhamnosus infection, treatment was changed to intravenous cefuroxime and erythromycin for three days. The extent of the consolidation and cavitation increased, respiratory and pulse rates rose, and he required oxygen therapy. The blood pressure fell $(80 / 50 \mathrm{~mm} \mathrm{Hg})$ and the serum urea concentration rose to $11.6 \mathrm{mmol} / \mathrm{l}$. He became exhausted and drowsy but not confused. Treatment was changed to intravenous gentamicin, 
Figure 2 Chest radiograph on the patient's eighth day in hospital showing extensive consolidation in the right upper and mid zones with relative sparing of the right lower zone.

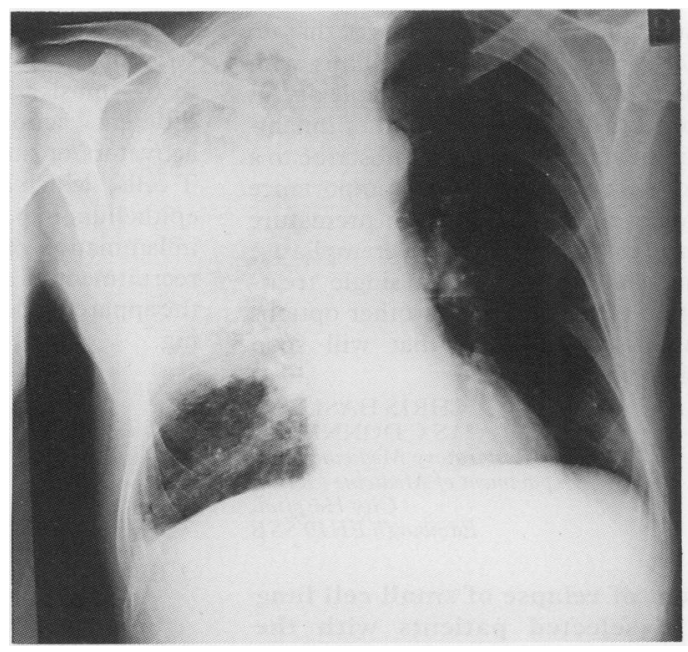

benzylpenicillin, flucloxacillin, and metronidazole for six days. He did not improve and despite a further change of treatment to intravenous ciprofloxacin and high dose cotrimoxazole for five days the patient died.

Necropsy showed extensive bronchopneumonia of the right lung and a large pus filled abscess cavity in the right upper lobe. Occasional Gram positive bacilli were identified, but special stains for fungi gave negative results. The left lung was oedematous. The heart was normal. Postmortem cultures of cardiac blood, lung abscess and tissue, and pleural fluid yielded only Gram positive bacilli similar in morphology and susceptibility patterns to the sputum isolates. Only sputum isolates were submitted to the Central Public Health Laboratory, Colindale, and the precise identification was given as $L$ casei ss rhamnosus.

\section{Discussion}

Only 25 cases of serious infections, mostly infective endocarditis, have been attributed to lactobacilli. ${ }^{3}$ Pneumonia with empyema due to lactobacilli has been described in only three cases, and in one of these ${ }^{1} L$ casei ss rhamnosus was the responsible microorganism. The portal of entry for the lactobacillus infection in our patient could have been the gastrointestinal tract or the oropharyngeal tract (or both) in view of the previously known reservoirs for this microorganism in man. ${ }^{4}$ Capsulated variants of $L$ casei ss rhamnosus were found to have increased virulence in experimental laboratory mice and rats. ${ }^{56}$

Lactobacilli are described as microaerophilic microorganisms that grow slowly in normal laboratory media, and their presence in clinical material may be missed. ${ }^{4}$ The use of several types of culture media with prolonged anaerobic incubation may increase the yield of pathogenic lactobacilli.

Our patient was treated with antibiotics to which the lactobacilli were susceptible in vitro yet there was only temporary improvement. This apparent discrepancy between the in vitro sensitivity of the organism and the response to appropriate therapeutic regimens was also noted in cases of lactobacillus endocarditis. ${ }^{7}$ Extensive in vitro studies ${ }^{8}$ suggest that in serious infections caused by lactobacilli synergistic bactericidal $\beta$ lactam-aminoglycoside combinations are the treatment of choice.

1 Rahman M. Chest infection caused by Lactobacillus casei ss rhamnosus. BMJ 1982;284:471-2.

2 Sutter VL, Citron DM, Edelstein MAC, Finegold SM, eds. Wadsworth anaerobic bacteriology manual. 4th ed. Belmonth, California: Star Publishing Company, 1985:129.

3 Bourne KA, Beebe JL, Lue YA, Ellner PD. Bacteraemia due to Bifidobacterium eubacterium, or Lactobacillus - twenty one cases. Yale J Biol Med 1978;51:505-12.

4 Kandler O, Weiss N. Genus Lactabacillus Beijerinck 1901, $212^{A L}$. In: Sneath PHA, Mair NS, Sharpe ME, Holt JG, eds. Bergey's Manual of systematic bacteriology. Vol 2. Baltimore: Williams and Wilkins, 1986:1209-34.

5 Sims WA. Pathogenic lactobacillus. J Pathol Bacteriol 1964; 87:99-105.

6 Rosan B, Hammond BF. Toxicity of Lactobacillus casei. Journal of Dental Research 1965;44:783-7.

7 Tenebaum MJ, Warner JF. Lactobacillus casei endocarditis. Ann Intern Med 1975;82:539.

8 Bayer AS, Chow AW, Concepcion NF, Guze LB. Comparative in vitro activity of five cephalosporins against lactobacilli. Antimicrob Agents Chemother 1979;16:112-3. 